\title{
Multiscale, thermomechanical topology optimization of cellular structures for porous injection molds
}

\author{
Tong Wu ${ }^{1}$, Kim Brand ${ }^{2}$, Doyle Hewitt ${ }^{3}$, Andres Tovar ${ }^{4}$ \\ ${ }^{1}$ Purdue University, USA, wu616@purdue.edu \\ 2 3D Parts Mfg, LLC, Indianapolis, USA, kim@3dpartsmfg.com \\ ${ }^{3}$ HEWITT MOLDING COMPANY, LLC, Kokomo, USA, Dhewitt@hewittmolding.com \\ ${ }^{4}$ Indiana University-Purdue University Indianapolis, Indianapolis, USA, tovara@iupui.edu
}

\section{Abstract}

During the injection molding cycle, molten material is injected at high pressure inside the mold and cooled down to form a solid part. This creates thermomechanical stresses that are alleviated by the correct design of a cooling system. In conventional molds, the cooling system consists of straight-line cooling channels, which can be manufactured using machining processes; however, they are thermally inefficient and unable to cool the injected part uniformly. The emergence of metal-based additive manufacturing techniques such as direct metal laser sintering (DMLS) allows the fabrication of molds with conformal cooling channels. Conformal cooling molds cool down the part faster and more uniformly; however, they face limitations. First, their fabrication cost is 10 to 20 times higher than the one of a conventional mold. Second, the DMLS process, which is the most popular fabrication method of conformal cooling molds, produces internal thermal stresses that distort the mold. The development of structural optimization methods such as multiscale topology optimization offers the potential to create novel and complex cellular structures that alleviate these current limitations. The objective of this research is to establish a multiscale topology optimization method for the optimal design of non-periodic cellular structures subjected to thermomechanical loads. The result is a hierarchically complex design that is thermally efficient, mechanically stable, and suitable for additive manufacturing. The proposed method seeks to minimize the mold mass at the macroscale, while satisfying the thermomechanical constraints at the mesoscale. The thermomechanical properties of the mesoscale cellular unit cells are estimated using homogenization theory. A gradient-based optimization algorithm is used for which macroscale and mesoscale sensitivity coefficients are derived. The design and evaluation of a porous injection mold is presented to demonstrate the proposed optimization method.

2. Keywords: 3D Multiscale topology optimization. Additive Manufacturing. Injection mold.

\section{Introduction}

Molds used in the plastic injection molding process must withstand extreme pressure loads and thermal expansion while at the same time providing dimensional accuracy of the molded part. These molds are required to efficiently and uniformly transfer heat flux from the molded part to cooling channels filled with running coolant [1]. Conformal cooling channels can be designed to provide uniform surface cooling, reduced injection mold cycle times and minimized part distortion. Additive Manufacturing (AM/3D Printing) enables the design and production of intricate conformal cooling channels in molds and mold inserts, offering significant cost savings, particularly in designs having high geometric complexity. These AM technologies include Direct Metal Laser Sintering (DMLS), Electron Beam Melting (EBM) and Laser Curing [2].

The unique capabilities of AM technologies allow an innovative design approach that challenges traditional guidelines of the plastics injection molding industry. Research has indicated significant advantages of AM generated, highly-complex conformal cooling channels. However, AM-generated conformal cooling channel face limitations. First, their fabrication cost is 10 to 20 times higher than the one of a conventional mold. Second, the DMLS process, which is the most popular fabrication method of conformal cooling molds, produces internal thermal stresses that distort the mold.

While much efforts aim to reduce the AM cost and improve the mold performance in manufacturing process [3], the development of structural optimization methods such as topology optimization offers the potential to create novel and complex injection mold designs with higher performance and reduced material cost [4]. However, traditional topology optimization method does not take contemporary manufacture 
limitations of AM technologies into account. In the resulting optimal design, undesired geometries such as small inclination, long overhangs, and thin bars may appear. To support these unstable structures, additional material would be required during AM process. As a result, the actual material usage is significantly increased.

Recently, many researches have investigated methods to bridging topology optimization and additive manufacturing [5]. These methods includes combining additional filters as constraints to improve the structure connectivity [6], and the utilization of topology optimization using functionally graded lattice structure $[7,8]$. The second method can be improved to a multiscale topology optimization methodology. By using this method, macroscale structure and mesoscale unit cells are able to be simultaneously optimized. Although this method has been an active research topic for many years [9, 10], few of them are applied this method in 3D and addressed manufacture issues. In this paper, a novel multiscale thermomechanical topology optimization approach is proposed, By employing this approach, a 3D porous injection mold is prototyped using self-supported unit cells with sufficient mechanical and thermal performance. The paper is organized as following: homogenization method will be briefly reviewed in section 4; Proposed multiscale thermomechanical topology optimization is presented in section 5; Section 6 provides test examples of the proposed method, and in section 7, a porous injection mold using this method is presented. Finally, conclusion is presented in section 8.

\section{Homogenization method}

The material design is displayed as a structural optimization problem and be optimized using inverse homogenization method [11]. The objective function of this method contains effective properties of investigated material, which are found by numerical homogenization. Numerical homogenization can be implemented in asymptotic method $(\mathrm{AH})$, mutual energy approach and represent volume element (RVE)-based approach . All of them can be used to derive homogenized elasticity tensor $\mathbf{D}_{c}^{H}$ and thermal conductivity tensor $\boldsymbol{\kappa}_{c}^{H}$ of an a-priori defined unit cell. In this section, these methods are briefly reviewed before presenting the proposed multiscale thermomechanical topology optimization approach.

\subsection{Asymptotic homogenization}

Asymptotic homogenization $(\mathrm{AH})$ assumes each mesoscale unit cell in a macroscale structure follows periodic boundary condition (PBC). The measurable quantity of a unit cell $u$ is the superposition of macroscale quantity $u_{0}(x, y)$ and a small periodically fluctuated mesoscale quantity $u_{1}(x, y)$, which can be represented using first order asymptotic expansion:

$$
u^{\epsilon}=u_{0}(x, y)+\epsilon u_{1}(x, y)+\mathcal{O}\left(\epsilon^{2}\right), \quad y=x / \epsilon, \quad \epsilon \ll 1 .
$$

Asymptotic homogenization can be rewritten in an equivalent discretized form in terms of element mutual energies:

$$
\mathbf{D}_{c}^{H}=\frac{1}{\left|V_{c}\right|} \sum_{e=1}^{n_{e}} \int_{V_{e}}\left[\mathbf{I}-\mathbf{B}_{e} \boldsymbol{\chi}_{e}\right]^{\top} \mathbf{D}_{e}\left[\mathbf{I}-\mathbf{B}_{e} \boldsymbol{\chi}_{e}\right] \mathrm{d} V_{e},
$$

where $n_{e}$ are the number of finite elements of the discretized unit cell, $\left|V_{c}\right|$ is the unit cell volume, $\mathbf{I}$ is the identity matrix, $V_{e}$ is the volume of the finite element $e, \mathbf{B}_{e}$ is the element strain-displacement matrix, $\mathbf{D}_{e}$ is the element elasticity tensor, and $\chi_{e}$ is the matrix containing the element displacement vectors $\chi_{e}^{i j}$ resulting from globally enforcing the unit test strain fields $\varepsilon^{i j}\left(\left[\chi_{e}^{11}, \chi_{e}^{22}, \chi_{e}^{12}\right]\right.$ for a 2D finite element). The element displacement vectors $\chi_{e}^{i j}$ are obtained from the global displacement vector of the unit cell $\chi_{c}^{i j}$, which is the solution of the equilibrium equation

$$
\left[\sum_{e=1}^{n_{e}} \int_{V_{e}} \mathbf{B}_{e}^{\top} \mathbf{D}_{e} \mathbf{B}_{e} \mathrm{~d} V_{e}\right] \chi_{c}^{i j}=\sum_{e=1}^{n_{e}} \int_{V_{e}} \mathbf{B}_{e}^{\top} \mathbf{D}_{e} \varepsilon^{i j} \mathrm{~d} V_{e} .
$$

The first term in the left hand side of Eq. (3) is the stiffness matrix of the unit cell and the right hand side is the nodal force vector of the unit cell.

In analogy to homogenization theory for elasticity tensor, homogenized thermal conductivity tensor $\boldsymbol{\kappa}_{c}^{H}$ of a discretized periodic unit cell is given by

$$
\boldsymbol{\kappa}_{c}^{H}=\frac{1}{\left|V_{c}\right|} \sum_{e=1}^{n_{e}} \int_{V_{e}}\left[\mathbf{I}-\mathbf{B}_{e}^{t} \mathbf{T}_{e}\right]^{\top} \boldsymbol{\kappa}_{e}\left[\mathbf{I}-\mathbf{B}_{e}^{t} \mathbf{T}_{e}\right] \mathrm{d} V_{e},
$$




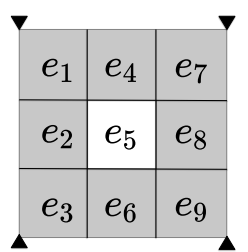

(a)

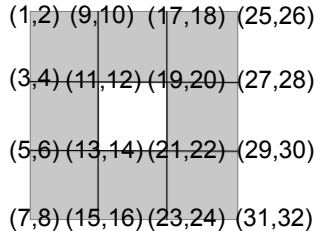

(b)

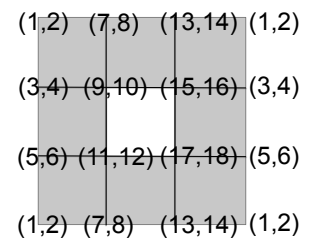

(c)

Figure 1: Comparison of Dofs setting between energy-based homogenization and asymptotic homogenization.(a) The discretized unit cell. (b) Dofs used in energy-based homogenization.(c) Dofs used in asymptotic homogenization.

where $n_{e}$ are the number of finite elements of the discretized unit cell, $\left|V_{c}\right|$ is the unit cell volume, $\mathbf{I}$ is the identity matrix, $V_{e}$ is the volume of the finite element, $\mathbf{B}_{e}^{t}$ is the element "strain" (temperature gradient)temperature matrix, $\boldsymbol{\kappa}_{e}$ is the element thermal conductivity tensor, and $\mathbf{T}_{e}$ is the matrix containing the element nodal temperature vectors $\mathbf{T}_{e}^{i j}$ resulting from globally enforcing the unit test temperature gradient fields ( $\left[\mathbf{T}_{e}^{1}, \mathbf{T}_{e}^{2}\right]$ for a $2 \mathrm{D}$ solid finite element). As before, the element temperature vectors $\mathbf{T}_{e}^{i}$ are obtained from the global temperature vector of the unit cell $\mathbf{T}_{c}^{i}$, which is the solution of the equilibrium equation

$$
\left[\sum_{e=1}^{n_{e}} \int_{V_{e}} \mathbf{B}_{e}^{t \boldsymbol{\top}} \boldsymbol{\kappa}_{e} \mathbf{B}_{e}^{t} \mathrm{~d} V_{e}\right] \mathbf{T}_{c}^{i}=\sum_{e=1}^{n_{e}} \int_{V_{e}} \mathbf{B}_{e}^{t \boldsymbol{\top}} \boldsymbol{\kappa}_{e} \mathbf{t}^{i} \mathrm{~d} V_{e} .
$$

The first term in the left hand side of Eq. (5) is the "stiffness" thermal matrix of the unit cell and the right hand side is the nodal heat flux vector of the unit cell.

\subsection{Energy-based homogenization}

Energy-based homogenization is an equivalent approach to asymptotic homogenization. In this method, the mutual energy form (Eq. (2) or (4)) and PBC are adopted as well. The difference between these two methods are the implementation of PBC and test strains. In energy-based homogenization, nodal displacement constraints are imposed on each pair of opposites boundaries $k-$ and $k+$ to satisfy PBC:

$$
\chi_{i}^{k+}-\chi_{i}^{k-}=\varepsilon_{0}^{i j} \Delta y
$$

where $\varepsilon_{0}^{i j}$ is a given strain and $\Delta y$ is the length of the unit cell [12]. In asymptotic homogenization, each pair of opposite boundaries share same Dofs (Fig.1) [13], and the test strains are imposed on the whole finite element.

\subsection{Representative volume element method}

Compared to above two methods, Representative volume element (RVE)-based method is straightforward. It is derived based on the assumption of constant strain fields are uniformly distributed over a RVE, thus homogenized elasticity tensor can be computed by average stress and strain using Hooke's law:

$$
\langle\boldsymbol{\sigma}\rangle=\langle\boldsymbol{E}\rangle\langle\varepsilon\rangle,
$$

where $\langle\boldsymbol{\sigma}\rangle$ is average stress, and $\langle\boldsymbol{\varepsilon}\rangle$ is average strain of a RVE. In finite element analysis, by applying a group of prescribed unit test strain on the RVE's boundaries, the homogenized properties can be obtained through computation of average stress of the whole element. Using the strain and displacement relations (for 2D problem):

$$
\varepsilon_{x}=\frac{\partial u}{\partial x}, \quad \varepsilon_{y}=\frac{\partial u}{\partial y}, \quad \gamma_{x y}=0.5 \times\left(\frac{\partial u}{\partial y}+\frac{\partial v}{\partial x}\right),
$$

applying prescribed displacement $\left[\begin{array}{lll}u=x & v=0\end{array}\right]$ on RVE's boundary yields an average stresses equal to $E_{1111}$ and $E_{2211}$, applying prescribed displacement $\left[u=0 \quad v=y\right.$ ] yields average stresses equal to $E_{2222}$ and $E_{1122}$, and applying prescribed displacement $[u=0.5 \times y \quad v=0.5 \times x]$ yields $E_{1212}$. 


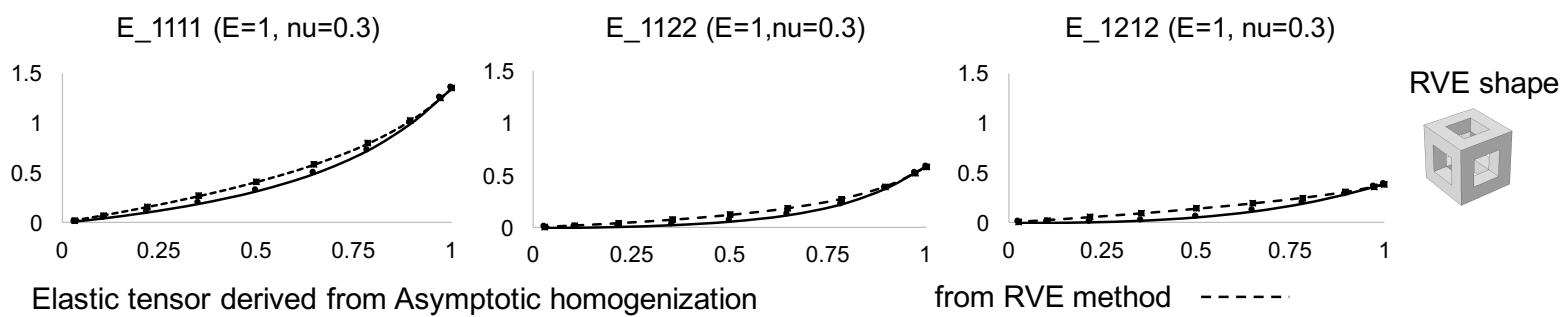

Figure 2: A comparison between RVE-based method and asymptotic homogenization method.

In analogy to this, homogenized thermal conductvity tensor can be computed by average heat flux and temperature gradient using Fourier's law:

$$
\langle\boldsymbol{q}\rangle=\langle\boldsymbol{\kappa}\rangle\langle\nabla \boldsymbol{T}\rangle
$$

where $\langle\boldsymbol{q}\rangle$ is average heat flux. It can be obtained by applying prescribed temperature $T_{1}=x$ and $T_{2}=y$ on boundaries.

To compare RVE and AH methods, a test using a group of 3D cubes with rectangular holes are implemented as Fig.2 shown. The resulting values from RVE-based method are slightly higher than those derived from $\mathrm{AH}$ method and the equivalent energy-based approach, which implies using RVE-based approach may over-evaluate stiffness of the structure. However, RVE-based method has several advantages over AH method. First, this method is appropriate to evaluate properties of non-periodic mesoscale material, because assumption of periodic boundary condition is not required, Second, Since prescribed displacements on the boundaries are linear functions of geometry coordinate, symmetry condition can be used for finite element analysis, if RVE's center located on the $(0,0,0)$. The computation cost will then be saved. Besides, this method can be conveniently implemented in many commercial softwares, which facilitate to homogenize a unit cell having complicate geometries.

\section{Multiscale thermomechanical topology optimization}

In this section, proposed multiscale thermomechanical topology optimization is presented. A flow chart (Fig. 3) is shown to describe this approach. First, a conceptual design is generated using macroscale topology optimization with a linear material interpolation. elemental strains and relative densities of this conceptual design are evaluated. Based on these information, each unit cell is optimized through RVEbased inverse homogenization. Then, homogenized tensor and local stiffness matrix of each optimal unit cells are computed through asymptotic homogenization $(\mathrm{AH})$. With assembled global stiffness matrix, the macroscale thermomechanical finite element analysis is performed to re-evaluated the objective. After these steps, the first iteration of multiscale optimization is finished.

The resulting porous structure from the first iteration has a weaker global stiffness than the conceptual design, because in conceptual design, the linear material interpolation represents a stiffer property than actual material with same densities. This leads to the re-evaluated objective worse than the value of conceptual design. Hence, design variables are updated by loosen the constraint functions and next iteration is performed. The approach contains three key concepts, namely macroscale structural design, mesoscale material design, and updating criteria, which will be described in following section.

\subsection{Macroscale structure design}

The purpose of marcoscale optimization is to use given mass, minimize the compliance arising from external load and thermal expansion, while remaining the molding and cooling qualities. The quality measurements include heat conduction and deformation of the molding part. Here, thermal compliance is adopted as a measurement of heat conduction, and displacements on surfaces of the molding part are used to evaluate the injected part deformation. These two measurements are employed as constraints. Additionally, In the optimization process, Hooke's and Fourier's law are served as physics constraints. 


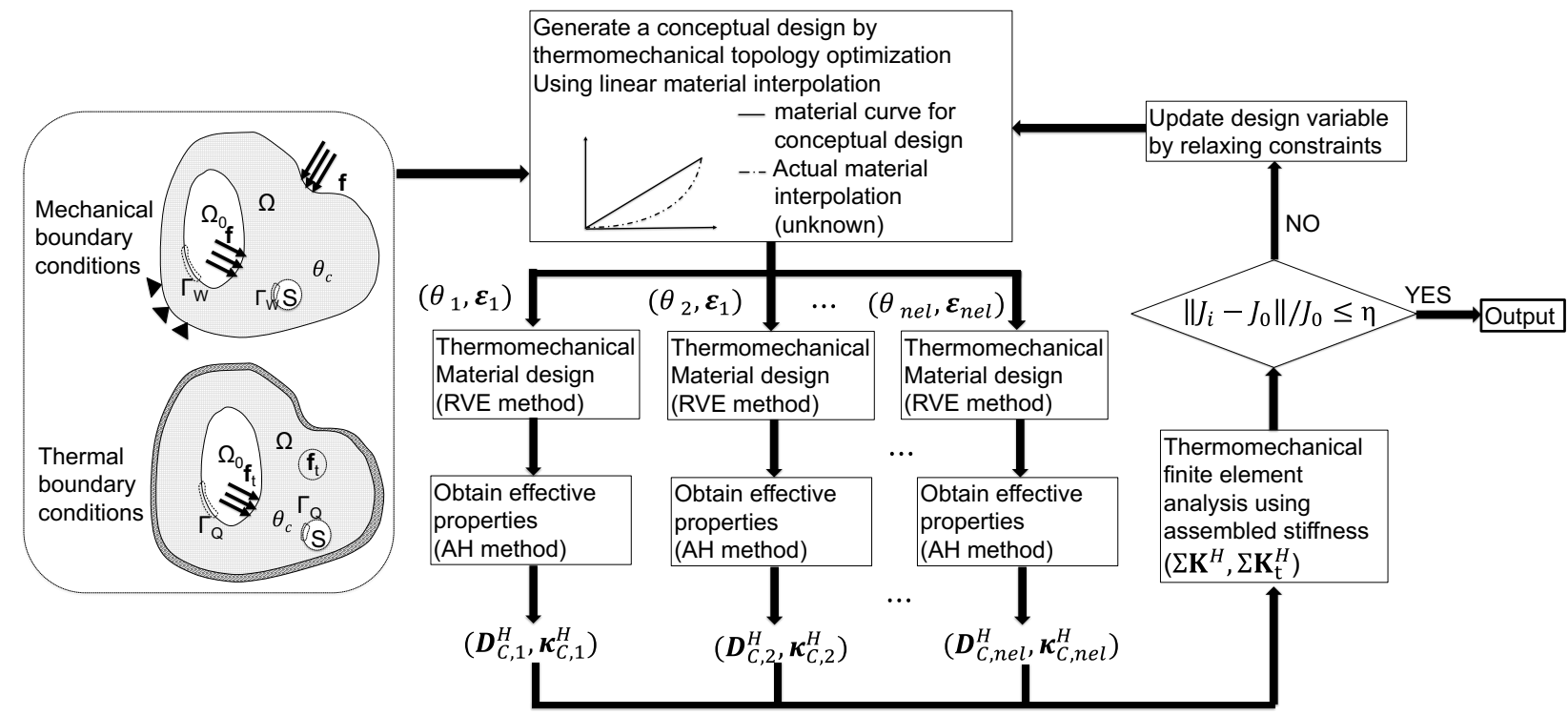

Figure 3: Flow chart of multiscale thermomechanical topology optimization.

Finally, macroscale thermomechanical topology optimization is stated as

$$
\begin{aligned}
\text { given } & m(\boldsymbol{\theta}) \\
\text { minimize } & \mathbf{f}^{\top} \mathbf{u}(\boldsymbol{\theta})+\mathbf{f}_{t}(\mathbf{T})^{\top} \mathbf{u}(\boldsymbol{\theta}) \\
\text { subject to } & m\left(\boldsymbol{\theta}_{\min }\right) \leq m(\boldsymbol{\theta}) \leq m\left(\boldsymbol{\theta}_{0}\right) \\
& \mathbf{q}^{\top} \mathbf{T}(\boldsymbol{\theta}) \leq C_{Q} \mathbf{q}^{\top} \mathbf{T}\left(\boldsymbol{\theta}_{0}\right) \\
& U_{j}(\boldsymbol{\theta})=\mathbf{1}_{j}^{\top} \mathbf{u}(\boldsymbol{\theta}) \leq C_{U} \mathbf{1}_{j}^{\top} \mathbf{u}^{\max }\left(\boldsymbol{\theta}_{0}\right), \quad j \in \Gamma_{W} \\
& \boldsymbol{\theta}^{\min } \leq \boldsymbol{\theta} \leq \mathbf{1} \\
\text { satisfying } & \mathbf{u}(\boldsymbol{\theta})=\mathbf{K}(\boldsymbol{\theta})^{-1} \mathbf{f} \\
& \mathbf{T}(\boldsymbol{\theta})=\left(\mathbf{K}_{t}(\boldsymbol{\theta})+\mathbf{K}_{h}\right)^{-1} \mathbf{q},
\end{aligned}
$$

where $\boldsymbol{\theta}$ represents relative density distribution and $\boldsymbol{\theta}_{0}$ is the initial design; $m$ is mass of macroscale structure; $\mathbf{f}$ is mechanical load and $\mathbf{f}_{t}$ is thermal expansion load; $\boldsymbol{u}$ is nodal displacement vector; $\mathbf{q}$ represents nodal heat flux and $\mathbf{T}$ nodal temperature. $U_{j}$ is the sum of nodal displacement, which equal to a vector $\mathbf{1}_{j}$ multiplies nodal displacement. $\mathbf{1}_{j}$ has value 1 at the location of molding part surface's degree of freedoms $\left(\Gamma_{W}\right)$, and 0 elsewhere. $\mathbf{K}$ is global stiffness matrix for mechanical; $\mathbf{K}_{t}$ is global stiffness for heat conduction, and $\mathbf{K}_{h}$ is constant global stiffness for heat convection. $C_{Q}, C_{U}$ are factors used to relax constraints.

The sensitivity analysis of this problem is described in [7]. Note that in design of an injection mold, the thermal expansion effect can be included in the injected pressure as a constant imposed on the molding part surface, therefore sensitivity of $\theta$ with respect to $\mathbf{f}_{t}$ is $\mathbf{0}$ in the optimization. In proposed multiscale approach, marcoscale topology optimization is only called once to generate a conceptual design. The design is generated using linear material interpolation, MMA solver and without filters.

\subsection{Material design using inverse homogenization}

By using the information provided by marcoscale conceptual design, extremed homogenized mechanical and thermal properties for each unit cell are found through inverse homogenization method. These properties mainly contain maximum bulk modulus $K$, maximum shear modulus $G$, and minimum Poisson ratio $\nu$. The first two properties are related to injection mold design. In addition, unit cell with maximum heat conduction requires to be investigated. In practice, the homogenized properties maximization problem can be rewritten as minimum compliance problem [14] and solved with PBC. Here, the compliance minimum problem is solved using RVE-based method. By applying elemental relative density $m\left(\boldsymbol{\theta}_{n e}\right)$, strain $\boldsymbol{\varepsilon}\left(\boldsymbol{\theta}_{n e}\right)$ and temperature gradient $\nabla \mathbf{T}\left(\boldsymbol{\theta}_{n e}\right)$ derived from marcoscale conceptual 
optimization, inverse homogenization is written as a minimum compliance problem. It is stated as a displacement based multiple load cases topology optimization:

$$
\begin{aligned}
\text { given } & m\left(\boldsymbol{\theta}_{n e}\right), \boldsymbol{\varepsilon}\left(\boldsymbol{\theta}_{n e}\right), \nabla \mathbf{T}\left(\boldsymbol{\theta}_{n e}\right) \\
\text { minimize } & \sum_{i=1}^{n_{1}} \mathbf{f}_{i}(\mathbf{u})^{\top} \mathbf{u}_{i}\left(m\left(\boldsymbol{\theta}_{n e}\right), \boldsymbol{\varepsilon}\left(\boldsymbol{\theta}_{n e}\right)\right) \\
\text { subject to } & m\left(\boldsymbol{\theta}_{\text {min }}\right) \leq m(\boldsymbol{\theta}) \leq m\left(\boldsymbol{\theta}_{n e}\right) \\
& \sum_{i=1}^{n_{2}} \mathbf{q}_{i}(\nabla \mathbf{T})^{\top} \mathbf{T}_{i}\left(m\left(\boldsymbol{\theta}_{n e}\right), \nabla \mathbf{T}\left(\theta_{n e}\right)\right) \leq C_{Q} \mathbf{q}^{\top} \mathbf{T}\left(\boldsymbol{\theta}_{n e}\right) \\
& \boldsymbol{\theta}^{\text {min }} \leq \boldsymbol{\theta} \leq \boldsymbol{\theta}^{\max } \\
\text { satisfying } & \mathbf{u}\left(\boldsymbol{\theta}_{n e}\right)=\mathbf{K}\left(\boldsymbol{\theta}_{n e}\right)^{-1} \mathbf{f} \\
& \mathbf{T}\left(\boldsymbol{\theta}_{n e}\right)=\left(\mathbf{K}_{t}\left(\boldsymbol{\theta}_{n e}\right)+\mathbf{K}_{h}\right)^{-1} \mathbf{q},
\end{aligned}
$$

where mechanical compliance (extreme elasticity property) is stated in the objective, thermal compliance (extreme heat conduction) is stated in the constraints. The prescribed displacement (or temperature gradient) and supports for each of them is defined in Fig. 4.

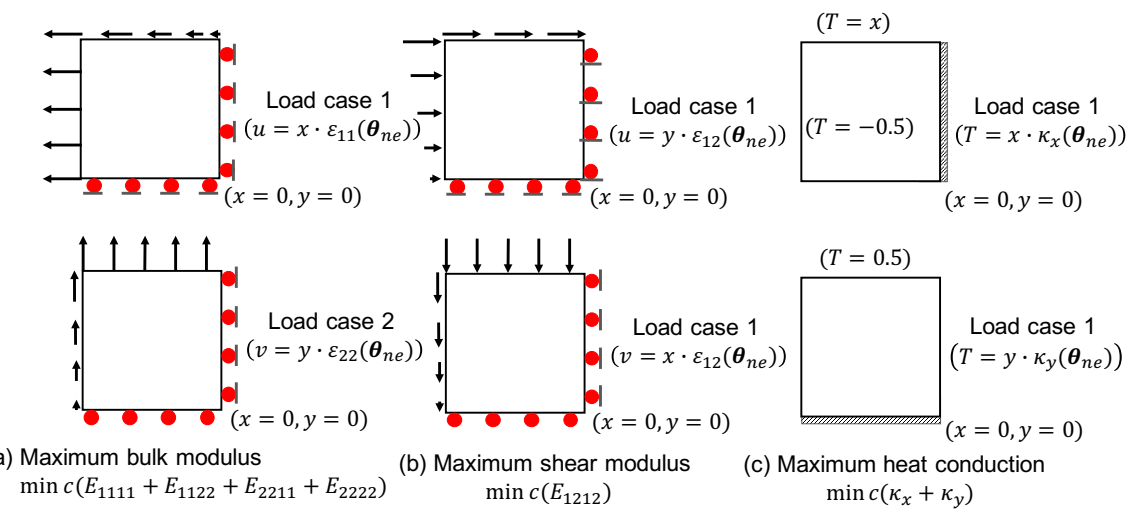

Figure 4: Prescribed displacement (or temperature gradient) and supports (or insulated boundary) for the purpose of maximizing extreme properties.

The problem is solved using SIMP method, MMA solver and density based filter with $m\left(\boldsymbol{\theta}_{n e}\right)=0.19$, 0.5 and 0.81 , prescribed strain $\boldsymbol{\varepsilon}\left(\boldsymbol{\theta}_{n e}\right)=(1,1,1)$, and prescribed temperature gradient $\nabla \kappa\left(\boldsymbol{\theta}_{n e}\right)=(1,1)$ (Fig. 5). Each unit cell is composed of $80 \times 80$ elements, but only a quarter of the structure $(40 \times 40$ elements) is required to be analyzed. The results are consistent with reference ([15],[16]), where PBC are assumed. With PBCs, an initial guess of uniform relative densities would result in fail of design variable updating. This can be avoided by using RVE method. In addition, as authors have tested, this method has better compatibility with a variety of initial guesses and filters.

$3 \mathrm{D}$ extension of this method is developed based on Top3d program [17]. Similarly, the problem is solved using SIMP method, MMA solver and density based filter with $m\left(\boldsymbol{\theta}_{n e}\right)=0.259,0.5$ and 0.74 , prescribed strain $\boldsymbol{\varepsilon}\left(\boldsymbol{\theta}_{n e}\right)=(1,1,1,1,1,1)$, and prescribed temperature gradient $\nabla \kappa\left(\boldsymbol{\theta}_{n e}\right)=(1,1,1)($ Fig. $6)$. The results are shown as a distribution of $2 \times 2 \times 2$ unit cells to illustrate the connectivity. Each unit cell is composed of $(40 \times 40 \times 40)$ elements, but only $1 / 8$ of the structure $(20 \times 20 \times 20)$ is required to be analyzed. To facilitate the removal of extra material cost in AM process, open channels are defined as passive elements, making $m\left(\boldsymbol{\theta}_{\max }\right)=0.8$.

By observation of results of extreme mechanical properties, it shows the result of maximum shear modulus is preferable for AM compared to max bulk modulus for its diamond-like self supporting structure. Therefore, it is selected as the mechanical objective for material design. Though this selection may not guarantee the overall material's properties, those properties can be achieved through design updating.

5.3. Updating criteria 


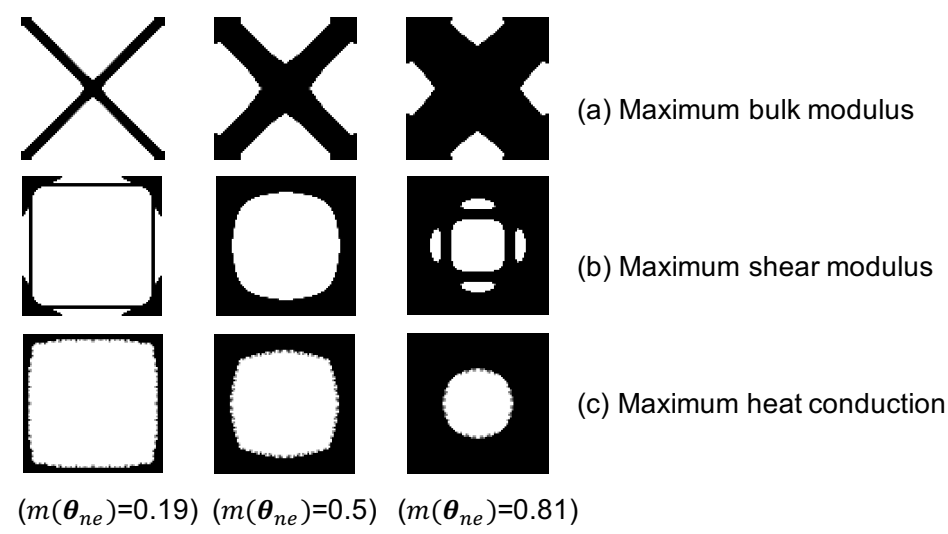

Figure 5: 2D results from RVE-based inverse homogenization.
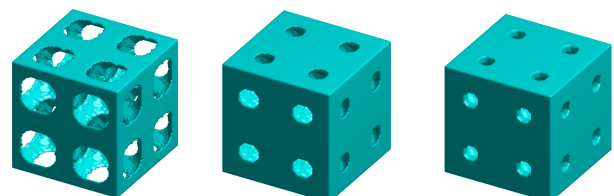

(a) Maximum bulk modulus
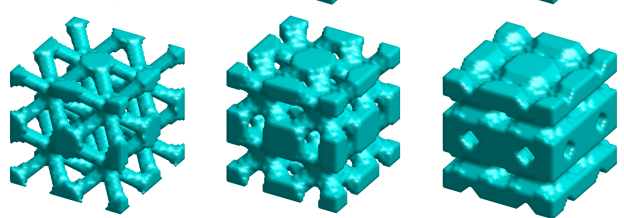

(b) Maximum shear modulus
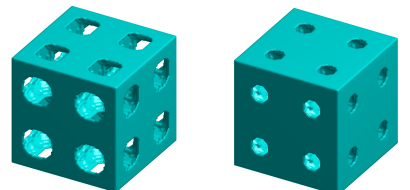

$\left(m\left(\boldsymbol{\theta}_{n e}\right)=0.259\right)$

$\left(m\left(\boldsymbol{\theta}_{\text {ne }}\right)=0.5\right)$

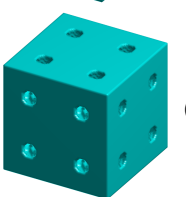

(c) Maximum heat conduction

$\left(m\left(\boldsymbol{\theta}_{n e}\right)=0.74\right)$

Figure 6: 3D results from RVE-based inverse homogenization.

After macroscale topology optimization and mesoscale material design for each unit cell, one iteration is finished. Next, the homogenized properties of each unit cell are evaluated through asymptotic homogenization. Then global stiffness matrices are assembled and a new objective value can be evaluated by calling thermomechanical finite element analysis. This value often worse than conceptual design, because in conceptual design a stiffer material interpolation scheme is used. To make a decision for updating, the convergence criteria is defined as:

$$
\eta_{i}=\left\|J_{i}-J_{0}\right\| / J_{0} \leq \eta,
$$

where $J_{i}$ is the objective evaluation of $i$ th iteration, $J_{0}$ is objective of conceptual design, $\eta$ is a small number. The following principle is employed to update elemental design variable $x_{i}$ or its Moore neighborhood $\mathcal{N}_{\mathcal{M}}:$

$$
\begin{aligned}
& \text { if } \quad J_{i, n e} \geq J_{0, n e} \quad \text { and } \quad x_{i, n e} \leq x_{\max }, \quad x_{i+1, n e}=x_{i, n e}+\delta \\
& \text { elseif } \quad J_{i, n e} \leq J_{0, n e} \quad \text { and } \quad x_{i, n e} \geq x_{\min }, \quad x_{i+1, n e}=x_{i, n e}-\delta \\
& \text { elseif } \quad J_{i, n e} \geq J_{0, n e} \quad \text { and } \quad x_{i, n e}=x_{\max }, \quad x_{i+1, \mathcal{N}_{\mathcal{M}}}=x_{i, \mathcal{N}_{\mathcal{M}}}+\delta \\
& \text { elseif } \quad J_{i, n e} \leq J_{0, n e} \quad \text { and } \quad x_{i, n e}=x_{\min }, \quad x_{i+1, \mathcal{N}_{\mathcal{M}}}=x_{i, \mathcal{N}_{\mathcal{M}}}-\delta
\end{aligned}
$$

Test examples are shown in following section to illustrate the performance presented method.

\section{Testing Examples}

This section presents $2 \mathrm{D}$ testing examples focusing on illustrating performance of proposed multiscale design approach. For the reader's convenience to compare the result with existing literature, two famous 
test examples, namely $\mathrm{MBB}$ and heat sink problem are used to optimize normalized mechanical and thermal compliance $C$ individually (Fig. 7 and 8). relative density $m\left(\boldsymbol{\theta}_{n e}\right)$ in macroscale is the design variable required to reflectively update. In mesoscale, max shear modulus is selected as the objective for mechanical problem, and max heat conduction for thermal problem. In addition, the examples use $\mathrm{AH}$ in mesoscale inverse homogenization with same initial guess are given for comparison (Fig. 9 and 10).

Coarse meshes are used in the examples, namely $15 \times 5$ for the MBB beam and $20 \times 20$ for the heat sink. Traditional topology optimization can neither use these coarse meshes to generate optimal black and white structures, nor prove to generate an optimal structure without undesired geometries for AM, which makes proposed multiscale approach effective.

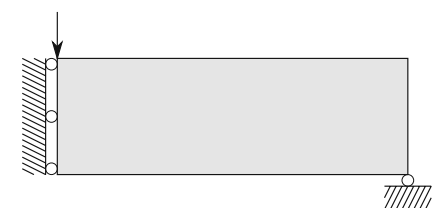

(a) The design domain, boundary conditions, and external load.

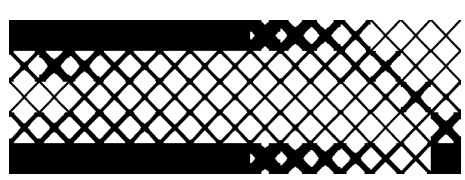

(c) Unit cell distribution after first iteration.

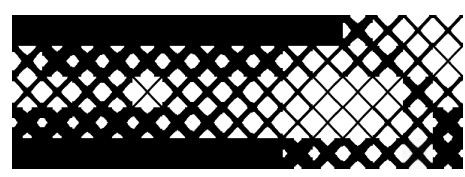

(e) Unit cell distribution after $20^{\text {th }}$ iteration.

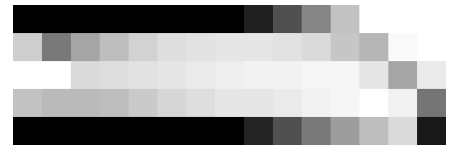

(b) Relative density distribution generated from conceptual design.

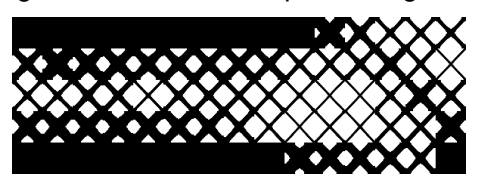

(d) Unit cell distribution after $10^{\text {th }}$ iteration.

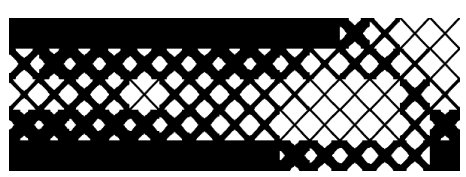

(f) Unit cell distribution after $28^{\text {th }}$ iteration. (g) Change of volume fraction
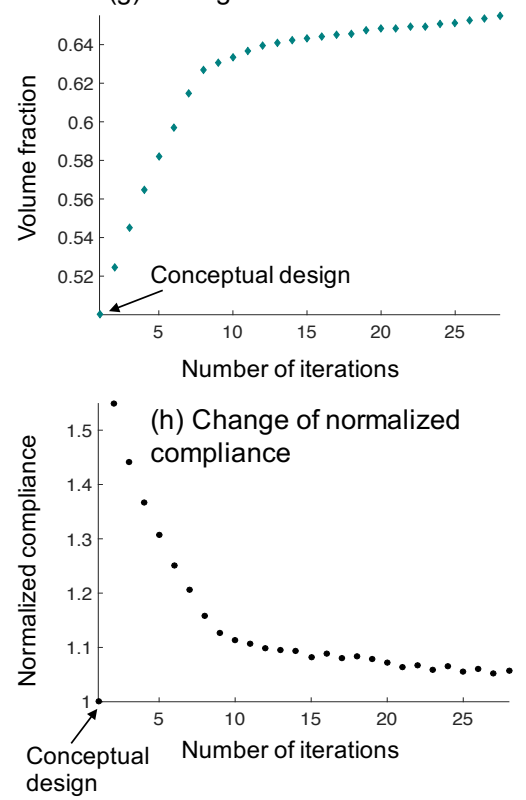

Figure 7: Minimize compliance of a MBB using proposed multiscale optimization. RVE method is implemented for material design.

30 iteration are performed for each example. All of these four examples show a convergence trend of $\eta$ and $m(\boldsymbol{\theta})$ with

$$
\delta=0.05 \times \eta_{i},
$$

though some fluctuation of $\eta$ appears in the optimization process of heat sink problem. From the results shown in above figures and Table 1, it is found that using RVE-based method for mesoscale material design is about two to three times faster than using AM method. In addition, it improves the connectivity of marcostructure and convergence.

Table 1: Key result evaluations of presented examples.

\begin{tabular}{|c|c|c|c|c|c|}
\hline Example & $C_{0}$ & $C$ & $m(\boldsymbol{\theta})$ & Element number & Computation time (s) \\
\hline 1 (Fig. 7) & 1.000 & 1.047 & 0.655 & 30000 & 741.9 \\
2 (Fig. 8) & 1.000 & 1.146 & 0.581 & 80000 & 2543.9 \\
3 (Fig. 9) & 1.000 & 1.069 & 0.651 & 120000 & 1377.3 \\
4 (Fig. 10) & 1.000 & 1.093 & 0.582 & 320000 & 6748.3 \\
\hline
\end{tabular}




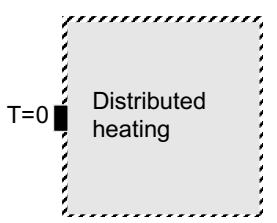

(a) The design domain, insulated boundaries, and heat sink.

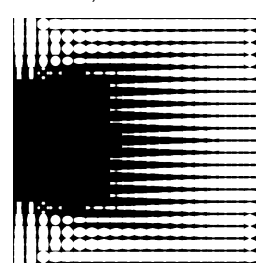

(c) Unit cell distribution after first iteration.

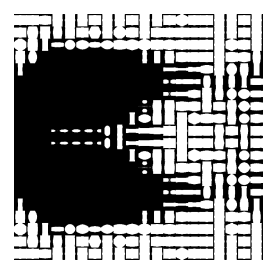

(e) Unit cell distribution after $20^{\text {th }}$ iteration.

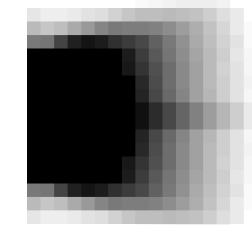

(b) Relative density distribution generated from conceptual design.

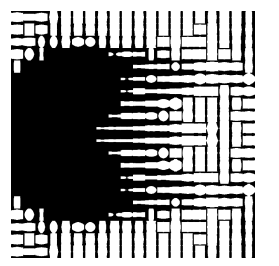

(d) Unit cell distribution after $10^{\text {th }}$ iteration.

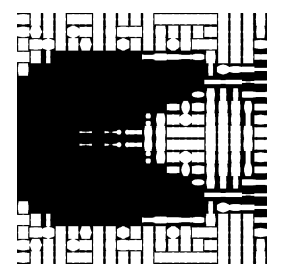

(f) Unit cell distribution after $28^{\text {th }}$ iteration. (g) Change of volume fraction
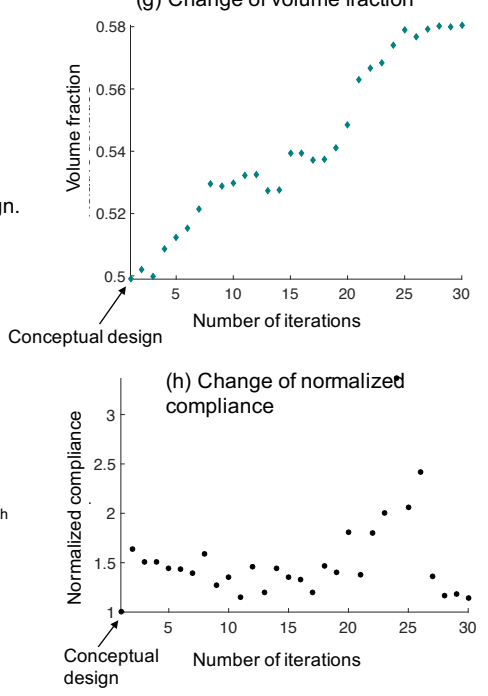

design

Figure 8: Minimize thermal compliance of a heat think using proposed multiscale optimization. RVE method is implemented for material design.

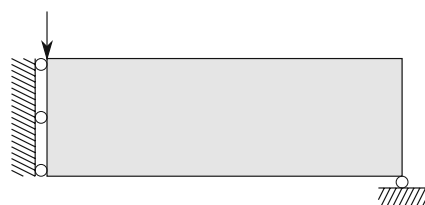

(a) The design domain, boundary conditions, and external load.

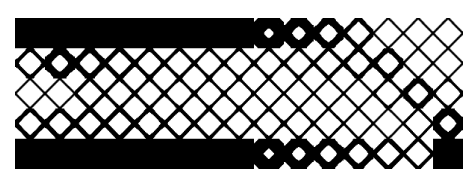

(c) Unit cell distribution after first iteration.

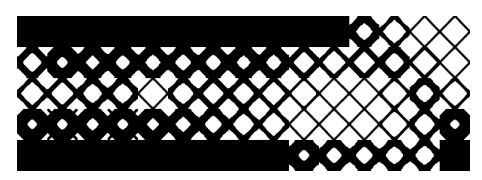

(e) Unit cell distribution after $20^{\text {th }}$ iteration.

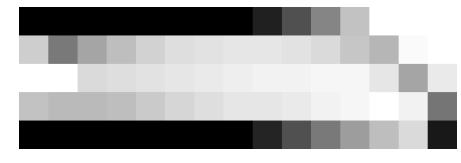

(b) Relative density distribution generated from conceptual design.

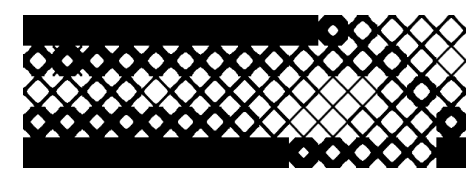

(d) Unit cell distribution after $10^{\text {th }}$ iteration.

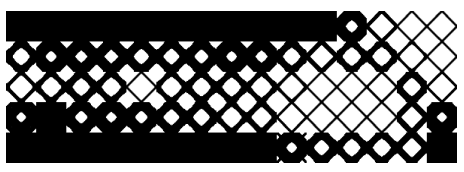

(f) Unit cell distribution after $30^{\text {th }}$ iteration. (g) Change of volume fraction

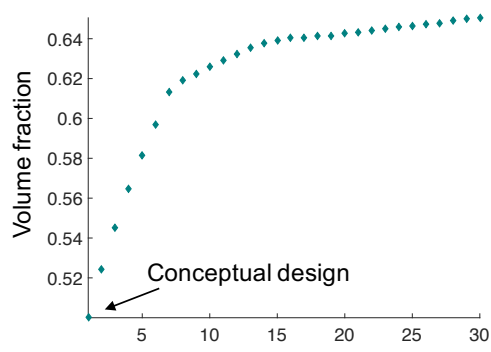

Number of iterations

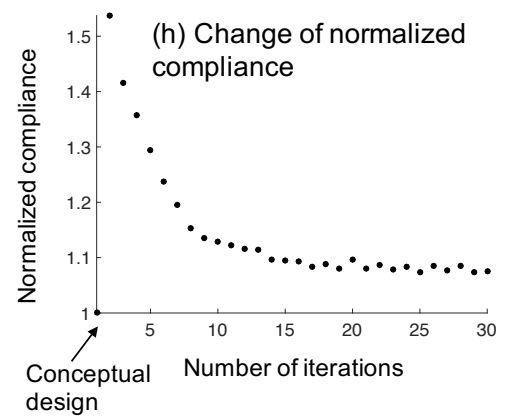

Figure 9: Minimize compliance of a MBB using proposed multiscale optimization. AH method is implemented for material design. 


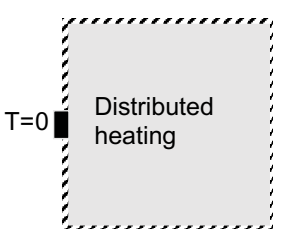

(a) The design domain, insulated boundaries, and heat sink.

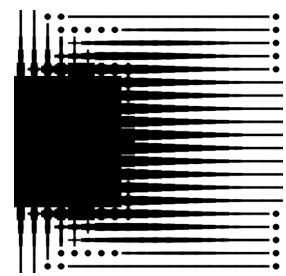

(c) Unit cell distribution after first iteration.

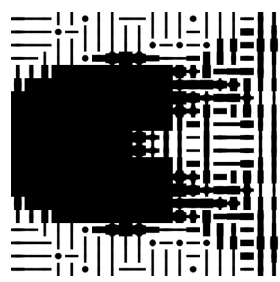

(e) Unit cell distribution after $20^{\text {th }}$ iteration.

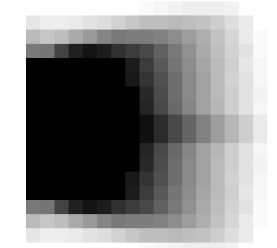

(b) Relative density distribution generated from conceptual design

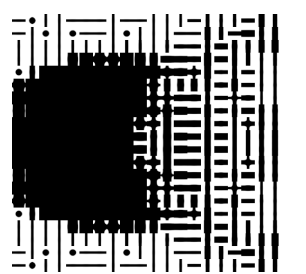

(d) Unit cell distribution after $10^{\text {th }}$ iteration.

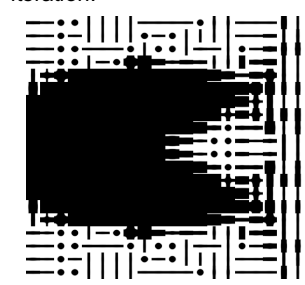

(f) Unit cell distribution after $28^{\text {th }}$ iteration.

(g) Change of volume fraction
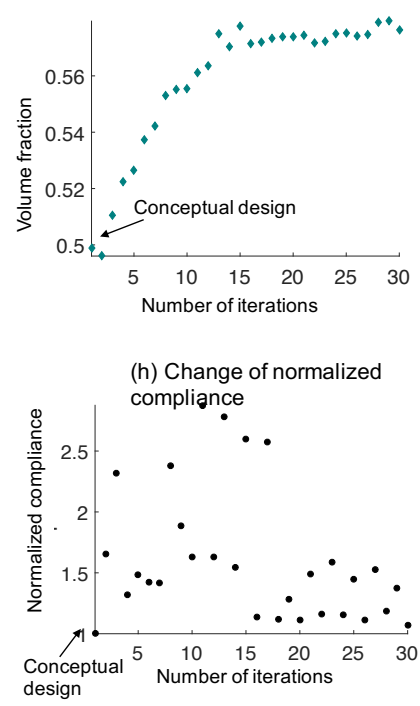

Figure 10: Minimize thermal compliance of a heat think using proposed multiscale optimization. AH method is implemented for material design.

\section{Application to porous injection mold}

A 3D porous injection mold design is presented in this section. The mold is a 3 in $\times 3$ in $\times 1.25$ in core insert. Based on the geometry of the mold, a quarter of mold section is investigated. Besides, the top core of the mold is reserved as solid structure for conformal cooling design (Fig. 11). Injection load located at the injected part surface, clamping pressure at imposed on bottom, and press-fit load on lateral sides are served as mechanical force. For heat conduction, a heat flux imposed on the injected part surface, and the temperature of cooling pipe is assumed as a constant value. All physics values are normalized in this problem.

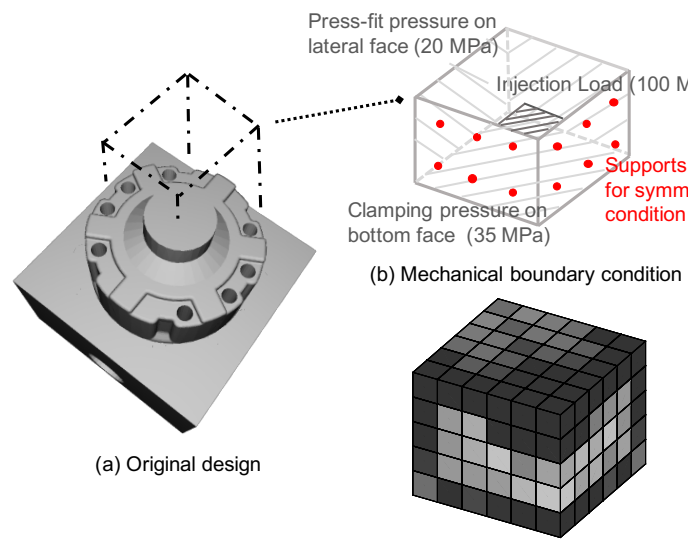

(d) Conceptual optimization

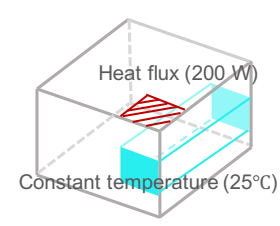

(c) Thermal boundary condition

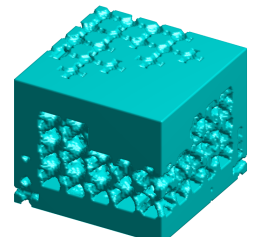

(e) Multiscale topology optimization

Figure 11: Multisclae thermomechanical topology optimization of a porous injection mold. 
In the problem statement, define given macroscale mass fraction $m(\theta)=0.5, C_{Q}=1.1, C_{U}=1.1, \eta=0.2$, $\delta=0.05 \times \eta_{i}$. A $6 \times 6 \times 5$ cubic mesh is applied to the macroscale problem, while a $20 \times 20 \times 20$ cubic mesh is used for each of mesoscale voxel. However, in mesoscale, only $1 / 8$ of each cubic is required to analyze. The convergence is satisfied after 15 iteration with $m(\boldsymbol{\theta})=0.71$. The optimal structure is approximated to an iso-surface and meshed to a STL file. The file size is $125.7 \mathrm{Mb}$, composed of 2511164 triangles. After modification in Netfabb, the triangle number is reduced to 374558, with a limit of deformation 0.01 in.

After mirroring the resulting section and performing boolean operations to assemble the top core and conformal cooling channel, the porous injection mold is prototyped using SLA printer FORMLAB 2+, with a scaled factor 0.4 (Fig. 12). It shows, only $10 \%$ more additional material is needed to support the structure. No support structure is required to change the orientation of the mold, and no internal structure is needed.

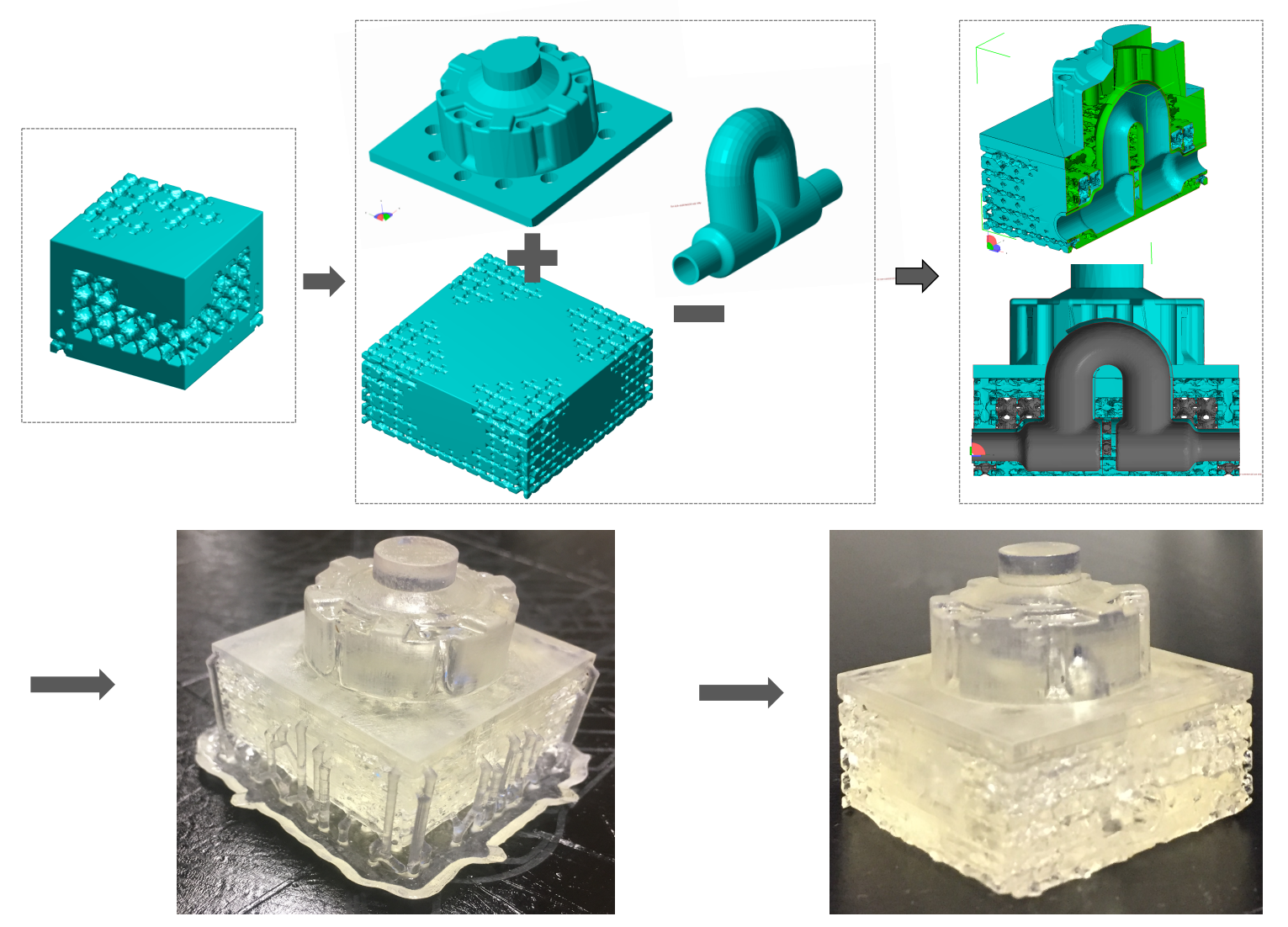

Figure 12: Assemble and prototyping process of the injection mold.

\section{Conclusion}

In this work, a practical multiscale topology optimization approach is presented. By considering both mechanical load and thermal conduction problem and extending the algorithm in $3 \mathrm{D}$, the method is suitable for injection mold optimization in industries. The using of RVE-based inverse homogenization in mesoscale significantly saves computational cost and improves the result. Limited by prevalent technology, the assembling operation capability in CAD would be strongly narrowed if the size and complexity of unit cell are increased. In the future work, a more efficient method for CAD post processing is worth to investigate.

\section{Acknowledgements}

The Walmart Foundation supported this research effort. Hewitt Molding Company provided the original injection mold model for the investigation. Any opinions, findings, conclusions, and recommendations expressed in this investigation are those of the writers and do not necessarily reflect the views of 
the sponsors.

\section{References}

[1] David O Kazmer. Injection mold design engineering. Carl Hanser Verlag GmbH Co KG, 2016.

[2] Lars-Erik Rännar, Anders Glad, and Claes-Göran Gustafson. Efficient cooling with tool inserts manufactured by electron beam melting. Rapid Prototyping Journal, 13(3):128-135, 2007.

[3] Antonio Armillotta, Raffaello Baraggi, and Simone Fasoli. Slm tooling for die casting with conformal cooling channels. The International Journal of Advanced Manufacturing Technology, 71(1-4):573$583,2014$.

[4] Tong Wu, Suchana A Jahan, Praveen Kumaar, Andres Tovar, Hazim El-Mounayri, Yi Zhang, Jing Zhang, Doug Acheson, Kim Brand, and Razi Nalim. A framework for optimizing the design of injection molds with conformal cooling for additive manufacturing. Procedia Manufacturing, 1:404$415,2015$.

[5] Tomas Zegard and Glaucio H. Paulino. Bridging topology optimization and additive manufacturing. Structural and Multidisciplinary Optimization, 2015.

[6] Quhao Li, Wenjiong Chen, Shutian Liu, and Liyong Tong. Structural topology optimization considering connectivity constraint. Structural and Multidisciplinary Optimization, 54(4):971-984, 2016.

[7] Tong Wu, Kai Liu, and Andres Tovar. Multiphase thermomechanical topology optimization of functionally graded lattice injection molds. In ASME 2016 International Design Engineering Technical Conferences and Computers and Information in Engineering Conference, pages V02AT03A036V02AT03A036. American Society of Mechanical Engineers, 2016.

[8] J Robbins, SJ Owen, BW Clark, and TE Voth. An efficient and scalable approach for generating topologically optimized cellular structures for additive manufacturing. Additive Manufacturing, 12:296-304, 2016.

[9] H Rodrigues, Jose M Guedes, and MP Bendsoe. Hierarchical optimization of material and structure. Structural and Multidisciplinary Optimization, 24(1):1-10, 2002.

[10] Liang Xia. Multiscale structural topology optimization. Elsevier, 2016.

[11] Ole Sigmund. Materials with prescribed constitutive parameters: an inverse homogenization problem. International Journal of Solids and Structures, 31(17):2313-2329, 1994.

[12] Liang Xia and Piotr Breitkopf. Design of materials using topology optimization and energy-based homogenization approach in matlab. Structural and multidisciplinary optimization, 52(6):1229-1241, 2015 .

[13] Erik Andreassen and Casper Schousboe Andreasen. How to determine composite material properties using numerical homogenization. Computational Materials Science, 83:488-495, 2014.

[14] M. P. Bendsøe and O Sigmund. Topology optimization: theory, method and applications. Springer, 2003.

[15] Ole Sigmund. A new class of extremal composites. Journal of the Mechanics and Physics of Solids, 48(2):397-428, 2000.

[16] MM Neves, H Rodrigues, and J Miranda Guedes. Optimal design of periodic linear elastic microstructures. Computers \& Structures, 76(1):421-429, 2000.

[17] Kai Liu and Andrés Tovar. An efficient 3d topology optimization code written in matlab. Structural and Multidisciplinary Optimization, 50(6):1175-1196, 2014. 